\title{
Long non-coding RNA PVT1 interacts with MYC and its downstream molecules to synergistically promote tumorigenesis
}

\author{
Ke Jin ${ }^{1,2,3}$. Shufei Wang ${ }^{2}$. Yazhuo Zhang ${ }^{2} \cdot$ Mengfang $\mathrm{Xia}^{2} \cdot$ Yongzhen $\mathrm{Mo}^{2} \cdot$ Xiaoling $\mathrm{Li}^{1,2,3} \cdot$ Guiyuan $\mathrm{Li}^{1,2,3}$. \\ Zhaoyang Zeng ${ }^{1,2,3} \cdot$ Wei Xiong ${ }^{1,2,3} \cdot$ Yi He $^{1,2}$
}

Received: 13 March 2019 / Revised: 22 June 2019 / Accepted: 5 July 2019 / Published online: 15 July 2019

(c) The Author(s) 2019

\begin{abstract}
Numerous studies have shown that non-coding RNAs play crucial roles in the development and progression of various tumor cells. Plasmacytoma variant translocation 1 ( $P V T 1$ ) mainly encodes a long non-coding RNA (lncRNA) and is located on chromosome 8q24.21, which constitutes a fragile site for genetic aberrations. PVT1 is well-known for its interaction with its neighbor $M Y C$, which is a qualified oncogene that plays a vital role in tumorigenesis. In the past several decades, increasing attention has been paid to the interaction mechanism between PVT1 and MYC, which will benefit the clinical treatment and prognosis of patients. In this review, we summarize the coamplification of $P V T 1$ and $M Y C$ in cancer, the positive feedback mechanism, and the latest promoter competition mechanism of PVTI and MYC, as well as how PVT1 participates in the downstream signaling pathway of c-Myc by regulating key molecules. We also briefly describe the treatment prospects and research directions of PVT1 and MYC.
\end{abstract}

Keywords PVT1 $\cdot$ MYC $\cdot$ Gene fusion $\cdot$ Positive feedback $\cdot$ Promoter $\cdot$ Enhancer

Abbreviations
$\begin{array}{ll}\text { PVT1 } & \text { Plasmacytoma variant translocation } 1 \\ \text { MYC } & \text { MYC or c-Myc } \\ \text { lncRNA } & \text { Long non-coding RNA } \\ \text { E-boxes } & \text { Enhancer box sequences } \\ \text { GWAS } & \text { Genome-wide association studies }\end{array}$

Ke Jin and Shufei Wang are equally contributed.

Wei Xiong

xiongwei@csu.edu.cn

$\triangle \mathrm{YiHe}$

yihe@csu.edu.cn

1 NHC Key Laboratory of Carcinogenesis (Central South University) and Hunan Key Laboratory of Translational Radiation Oncology, Hunan Cancer Hospital and the Affiliated Cancer Hospital of Xiangya School of Medicine, Central South University, Changsha, Hunan, China

2 The Key Laboratory of Carcinogenesis and Cancer Invasion of the Chinese Ministry of Education, Cancer Research Institute, Central South University, Changsha, Hunan, China

3 Hunan Key Laboratory of Nonresolving Inflammation and Cancer, Disease Genome Research Center, The Third Xiangya Hospital, Central South University, Changsha, Hunan, China
SNPs Single nucleotide polymorphisms

TAD Topologically associated domain

BRD4 Bromodomain-containing protein 4

CDK4 Cyclin-dependent kinases 4

Miz1 Myc-interacting zinc finger protein-1

Snail1 Snail homolog 1

TFAP4 Transcription factor AP4

EZH2 Enhancer of zeste homolog 2

TSP1 Thrombospondin-1

VEGF Vascular endothelial growth Factor

HIF-1 Hypoxia inducible factor-1

LDH Lactate dehydrogenase

GLUT1 Glucose transporter 1

HK2 Hexokinase 2

PDK Pyruvate dehydrogenase kinase

MDM2 Mouse double minute 2

\section{Introduction}

In the entire human genome, only $2 \%$ of the genes are used to encode proteins, and the vast majority of the human genome is transcribed into non-coding RNAs, such as long non-coding RNAs (lncRNAs), which are longer than 200 nucleotides (nt) [1]. These lncRNAs play an important role 
in regulating cellular activities by modulating gene expression, including cell differentiation, proliferation, cell cycle, apoptosis, migration, and invasion [2, 3]. However, abnormal expression of IncRNAs can contribute to the occurrence and development of various diseases, such as cancer [4-6].

The plasmacytoma variant translocation 1 ( $P V T 1)$ gene encodes a lncRNA and was first discovered as an activator of $M Y C$ in murine plasmacytoma variant translocation in 1984 [7]. The human $P V T 1$ is a large locus more than $30 \mathrm{~kb}$ in length and is located at 8q24.21 [8]. The locus constitutes a fragile site for genetic aberrations, including translocation, amplification, viral integration, and multiple risk loci in cancer or other diseases $[8,9]$.

Studies have shown that PVT1 is expressed at low levels in normal tissues, while it is highly expressed in various malignant tumors and tumor cell lines, such as gastric cancer, lung cancer, hepatocellular carcinoma, thyroid carcinoma, breast cancer, and pancreatic cancer [7, 9-16]. PVT1 can also serve as a potential predictor of cancer progression and patient prognosis [10, 14, 15]. Most studies have reported that PVT1 can promote proliferation, angiogenesis, apoptosis escape, and participate in DNA rearrangements, which might ultimately promote carcinogenesis $[7,10$, $11,13,17,18]$. However, recent studies have shown that $P V T 1$, also a microRNA Host gene, can encode miR-1204, miR-1205, miR-1206, miR-1207-5p, miR-1207-3p, and miR-1208 [7, 19-21]. Moreover, PVT1 can also engender a circRNA called circPVT1 by circularizing exon 3 along with long introns on each side [22]. CircPVT1 can act as a miRNA sponge to regulate gene expression and promote cell proliferation in cancer [22, 23].

In the human genome, $M Y C$ is located only $53 \mathrm{~kb}$ upstream of $P V T 1$. Both genes have been reported to play a role in cancer [1]. High expression of PVT1 can increase c-Myc expression by regulating c-Myc stability, and they can also interact with each other to regulate their expression, which synergistically promotes the occurrence and development of tumors [7, 24]. MYC, a proto-oncogene, was first discovered as the cellular homolog of the Avian virus myelocytomatosis oncogene and plays various roles in protein synthesis, metabolism, and cellular differentiation $[25,26]$. C-Myc, a transcription factor, is thought to regulate the expression of $15 \%$ of all genes by binding enhancer box sequences (E-boxes) [27]. Moreover, c-Myc can lead to genomic instability, gene amplification, cellular proliferation, and repression of apoptosis, which is observed in various tumors, including breast, lung, colon, and prostate cancers $[25,27]$. Although numerous studies have examined the interaction of PVT1 and MYC, the detailed mechanisms of the interaction between them remain unclear. In this review, we update the more recent findings for PVT1 and MYC, with the aim of promoting modern studies and the development of clinical therapies.

\section{Cancer risk related to PVT1 fusion gene on 8q24}

Both human PVTI and MYC are located at the 8q24 locus, a well-known cancer-associated chromosome segment and a common and preferred integration site for somatic cell expansion in many cancers (Fig. 1), such as prostate, colorectal, breast, ovarian, and cervical cancers [28-32]. The fragile sites are heritably specific chromosomal loci. When cells are undergoing mitosis, the chromatin region exhibits a loose state and high DNA flexibility, and the induction of fragile sites inhibits partial replication without blocking the cell cycle [33]. There are two common fragile sites in the 8q24 segment, FRA8C and FRC8D, where substantial amounts of DNA helix flexibility exist. When cells are under

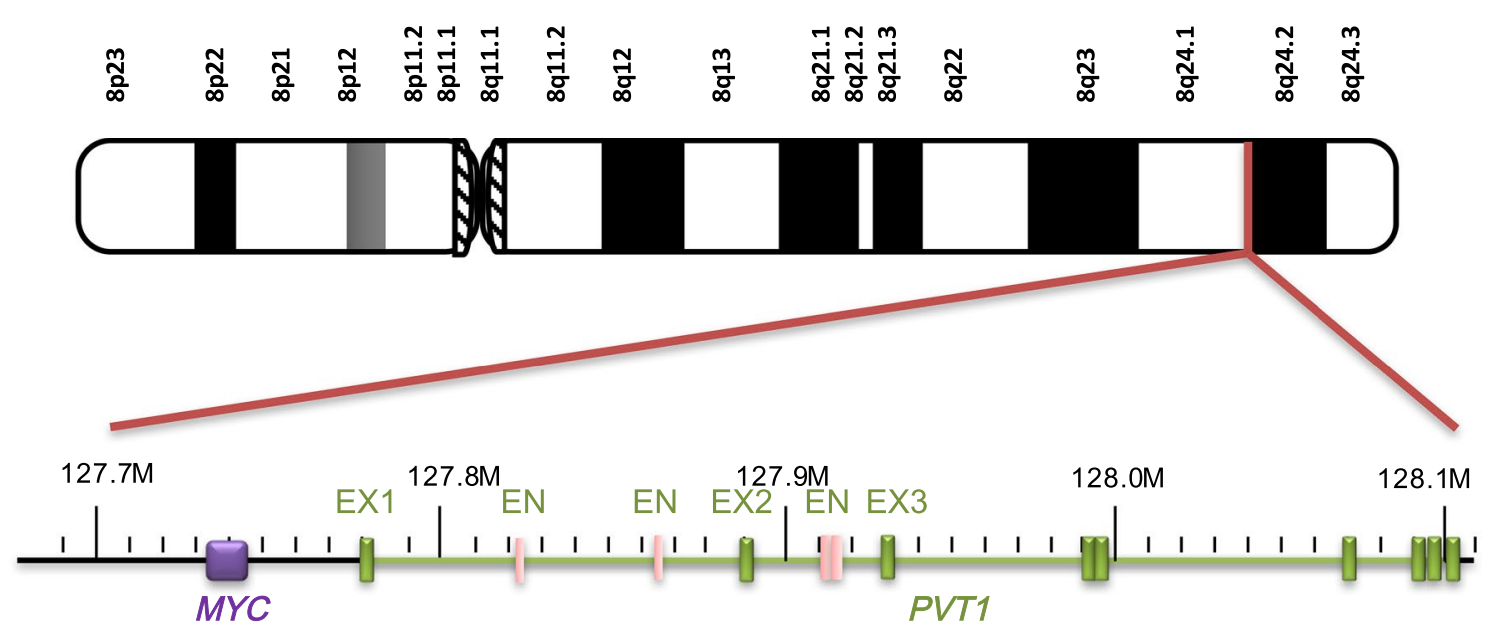

Fig. 1 Location of $P V T 1$ and $M Y C$ on 8q24. EX exon, $E N$ enhancer 
the influence of genetic changes or exposed to factors that interfere with DNA replication, such as hypoxia, viral invasion, and cytotoxic drugs, these sites induce chromosome breakage and amplification, translocation, and other chromosome structural variations [34]. As a gene sequence adjacent to the fragile site, $P V T l$ is also prone to gene rearrangement within the chromosome or interchromosomal segments during gene amplification, leading to an increase in its copy number. Cytogenetic analysis indicated that gene amplification was driven by recurrent breakage within the common fragile site via a break-fusion-bridge mechanism $[35,36]$. Although no studies have specifically reported the mechanism of the increased $P V T 1$ copy number, we predict that its amplification occurs through this general amplification mechanism at the fragile sites.

Amplification of the PVTI locus often manifests as the fusion of sister chromatid ends to form double minute chromosomes or a homogeneously staining region [36]. Through either amplification process, $P V T l$ will generate new fusion genes, and these abnormal changes in the gene replication process play an important role in tumor development [37]. Numerous studies have shown that $P V T l$ can fuse to other genes by translocation in certain tumor tissues (Table 1). Although $P V T 1$ is a nonprotein encoding gene, the fusion of other genes may produce abnormal protein that participates in the cancer process. For example, in small cell lung cancer, exon 1 or 2 of $P V T 1$ fuses to exon 2 of $A K T 3$ (1q44) to form a chimeric transcript $P V T 1-A K T 3$ [38]. It encodes a shorter AKT3 protein with a missing $\mathrm{N}$-terminus and an incomplete $\mathrm{PH}$ domain, which changes the function of the protein and promotes tumor progression [38]. We also found that gene fusion of $P V T l$ with its neighboring gene $M Y C$ is most common in 8q24-amplified cancers [37, 39]. An increased $M Y C$ copy number and $P V T 1$ expression occur in more than $98 \%$ of cancer cases with increased 8q24 copy numbers, which points to an interaction between $P V T 1$ and $M Y C$ and that they are part of a common signaling pathway [40]. False DNA repair, referred to as chromothripsis, may occur during amplification of the $P V T 1$ locus, producing fragmented 8q24 amplicons. In different tumors, these fragments, including $P V T 1$ and $M Y C$, fuse with different exons [39]. Thus, $M Y C$ can be regulated by the $P V T 1$ promoter because of the loss of its partial exons [39]. In mice artificially transfected with copies of MYC-PVT1, the level of RSPO1, a key regulatory molecule in the $\mathrm{Wnt} / \beta$-catenin pathway, was upregulated. Accelerating proliferation of breast cancer cells in mice was subsequently observed, and the increased copy number of each single gene separately did not have a proliferative effect [41]. Therefore, most studies have shown that $P V T 1$ fusion genes exert the effect of affecting cancer risk mainly by driving protein expression. With respect to whether it affects the function of lncRNA, additional research is expected.

From the perspective of genetic epidemiology, genomewide association studies (GWAS) indicate that 8q24 is a cancer susceptibility locus and single nucleotide polymorphisms (SNPs) in this region are associated with cancers, such as colorectal, prostate, breast, ovarian, and pancreatic cancers [52-58]. The cancer risk-associated SNPs are differentially located on 8q24 in different cancers; for example, rs 1561927 in pancreatic cancer locates $455 \mathrm{~kb}$ telomeric of
Table 1 Fusion genes of $P V T 1$ in malignancy

\begin{tabular}{llll}
\hline Cancer type & Fusion locus & Fusion gene & References \\
\hline Colorectal cancer & $8 \mathrm{q} 24 / 8 \mathrm{q} 23.1$ & $P V T 1-R S P O 2$ & {$[42]$} \\
Neuroendocrine bladder cancer & $8 \mathrm{q} 24 / 8 \mathrm{q} 24.21$ & $P V T 1-$ MYC & {$[37]$} \\
Acute myeloid leukemia & $8 \mathrm{q} 24 / 17 \mathrm{q} 12$ & $P V T 1-E R B B 2$ & {$[43]$} \\
& $8 \mathrm{q} 24 / 8 \mathrm{q} 24.21$ & $P V T 1-C C D C 26$ & {$[44]$} \\
Gastric cancer & $8 \mathrm{q} 24 / 8 \mathrm{q} 24.13$ & $P V T 1-N S M C E 2$ & {$[45,46]$} \\
& $8 \mathrm{q} 24 / 11 \mathrm{p} 13$ & $P V T 1-P D H X$ & {$[47]$} \\
& $8 \mathrm{q} 24 / 10 \mathrm{q} 26.13$ & $P V T 1-A T E 1$ & {$[47]$} \\
Blastic plasmacytoid dendritic cell & $8 \mathrm{q} 24 / 11 \mathrm{p} 13$ & $P V T 1-A P I P$ & {$[47]$} \\
neoplasm & $8 \mathrm{q} 24 / 10 \mathrm{q} 26.12$ & $P V T 1-P P A P D C 1 A$ & {$[47]$} \\
Burkitt lymphoma & $8 \mathrm{q} 24 / 6 \mathrm{p} 21$ & $P V T 1-S U P T 3 H$ & {$[48]$} \\
Small-cell lung cancer & & & {$[49]$} \\
& $\mathrm{t}(2,8) \mathrm{or} \mathrm{t}(8,22)$ & $P V T 1-I G \gamma$ or IGk & {$[50]$} \\
& $8 \mathrm{q} 24 / 8 \mathrm{q} 13.3$ & $P V T 1-E Y A 1$ & {$[51]$} \\
Multiple myeloma & $8 \mathrm{q} 24 / 8 \mathrm{q} 12$ & $P V T 1-C H D 7$ & {$[38]$} \\
Medulloblastoma & $8 \mathrm{q} 24 / 1 \mathrm{q} 44$ & $P V T 1-A K T 3$ & {$[38]$} \\
& $8 \mathrm{q} 24 / 13 \mathrm{q} 13$ & $P V T 1-N B E X$ & {$[38]$} \\
\hline
\end{tabular}


PVTl, while rs 10088218 in ovarian cancer lies $400 \mathrm{~kb} 3^{\prime}$ of $M Y C$ [56-59]. Furthermore, it has been shown that the $M Y C$ allele in cis linked to the cancer risk-associated SNP variant shows significantly higher expression than the $M Y C$ allele linked to the nonrisk-associated variant [60]. The GWAS study in colorectal cancer samples and normal tissue control samples found that SNP rs6983267 on 8q24 is a transcriptional enhancer [61]. Moreover, the chromosome conformation capture assay demonstrated that the risk region interacted with $M Y C$ in the long-range physical way, leading to tumor development [61]. At the same time, chromosome conformation capture research also indicated that rs6983267 has interactions with the adjacent $P V T 1$ promoter $[60,62]$. Moreover, rs378854 can also interact with $M Y C$ or $P V T 1$ promoter in prostate cancer [63]. The 8q24 region contains multiple independent risk regions of prostate cancer, colon cancer, breast cancer, and pancreatic cancer [64, 65], all of which have been shown to affect distant target genes and thereby increase cancer susceptibility. These risk regions exhibited strong interactions with $M Y C$ in their respective cancer cell lines, whereas no risk SNP transcription associated with chr8 transcription was found in normal tissues. These observations provide genetic statistical evidence that PVTI and MYC are relevant to cancer risk at the chromosome level.

\section{Positive feedback between PVT1 and MYC}

Since $P V T 1$ was discovered and received widespread attention, researchers have closely examined whether it is involved in the cancer-promoting pathway related to MYC. A large number of studies showed that PVT1 levels are higher in many cancers with increased MYC expression compared to normal tissues and are associated with poor prognosis [66-68]. Tissue microarray analysis of 8 primary tumors (lung, colon, rectum, stomach, esophagus, liver, kidney, and mammary gland) indicated a high correlation between PVT1 and c-Myc expression, providing strong evidence for the cooperation of PVT1 and MYC in different human cancers [40]. The gene fusion of $M Y C$ and $P V T 1$ and the positive feedback mechanism between them can increase the transcription of PVT1, thereby enhancing the role of PVT1 in tumors.

Interestingly, when siRNA was used to knock down PVT1, low levels of c-Myc were found without significant changes in MYC mRNA levels, which indicates that c-Myc levels are closely related to PVT1 in high-copy 8q24 proliferating cancer cells. Phosphorylation of the threonine 58 (Thr58) site on c-Myc causes the degradation of itself through the ubiquitin proteasome pathway [69]. In tumor tissues, the major transcription product of the PVTI gene, PVT1 (an lncRNA), increases the level of c-Myc by enhancing the stability of it via blocking the phosphorylation of the Thr58 site on c-Myc [8, 70, 71]. Furthermore, c-Myc is an important transcription factor that enhances PVT1 transcription. Carramusa et al. [72] found that the PVT1 promoter region contains two enhancer E-boxes that serve as c-Myc binding sites and E-box 2 clearly mediates the binding of c-Myc to the $P V T 1$ promoter to promote PVT1 expression. Northcott et al. [39] subsequently confirmed that MYC positively regulates the expression of $P V T 1$-encoded miRNA in medulloblastoma cells and $M Y C$ can enhance its own expression through the $P V T 1$ promoter in tumors with PVT1-MYC gene fusion. Thus, the mechanism of coamplification of $P V T 1$ and $M Y C$ in tumor cells may involve a positive feedback pathway in which c-Myc increases the transcription of PVT1 by binding to E-boxes located in the PVTI promoter region, which results in the increased expression of PVT1. PVT1 then prevents the degradation of c-Myc by blocking the phosphorylation of Thr58 in c-Myc and maintains a high c-Myc protein level (Fig. 2). Therefore, through this positive feedback mechanism, PVT1 and c-Myc in cancer cells can remain at a high level, which results in the synergistic promotion of tumorigenesis by PVT1 and c-Myc.

\section{Promoter-enhancer competition between PVT1 and MYC}

It has been known for many years that enhancers play an important role in genomic transcription. Their main function is to enhance the transcriptional initiation rate as a sequence element to enhance promoter activity. Initially, the understanding of enhancer-promoter interactions only existed at the linear level. With the development of high-throughput techniques and chromosome topology spatial structure detection techniques, researchers were able to detect the interaction among genomic components in $3 \mathrm{D}$ and discover more complex mechanisms [73, 74]. There are two mechanisms for the spatial interaction between the promoter and enhancer. One mechanism is to connect linearly distant loci with proteins, such as CTCF. In the other mechanism, for the topologically associated domain (TAD) scaffold, contact between the promoter and enhancer can coordinate the regulation of gene expression [75, 76]. Promoter-enhancer competition between $P V T 1$ and $M Y C$, described as follows, mainly occurs in the TAD.

Cho et al. [9] found that the PVT1 promoter can ciscompetitively contact its own four intragenic enhancers, reducing competition from the $M Y C$ promoter and downregulating the expression of $M Y C$ to inhibit the proliferation of tumor cells. Unexpectedly, they found that when the PVT1 promoter was silenced, tumor cell proliferation was significantly enhanced, which was contrary to the experimental conclusion from previous studies that " $P V T 1$ has a 
Fig. 2 Positive feedback between PVT1 and MYC. C-Myc protein tends to be phosphorylated at the Thr-58 site by GSK $3-\beta$ and then degraded by proteasome. However, PVT1 promotes c-Myc stability by inhibiting the degradation and phosphorylation of the Thr-58 site of c-Myc. Thus, c-Myc can return to the nucleus to interact with the $P V T 1$ promoter, leading to the upregulation of PVT1. PVT1, the transcription product of $P V T 1$, joins the positive feedback pathway to stabilize c-Myc protein

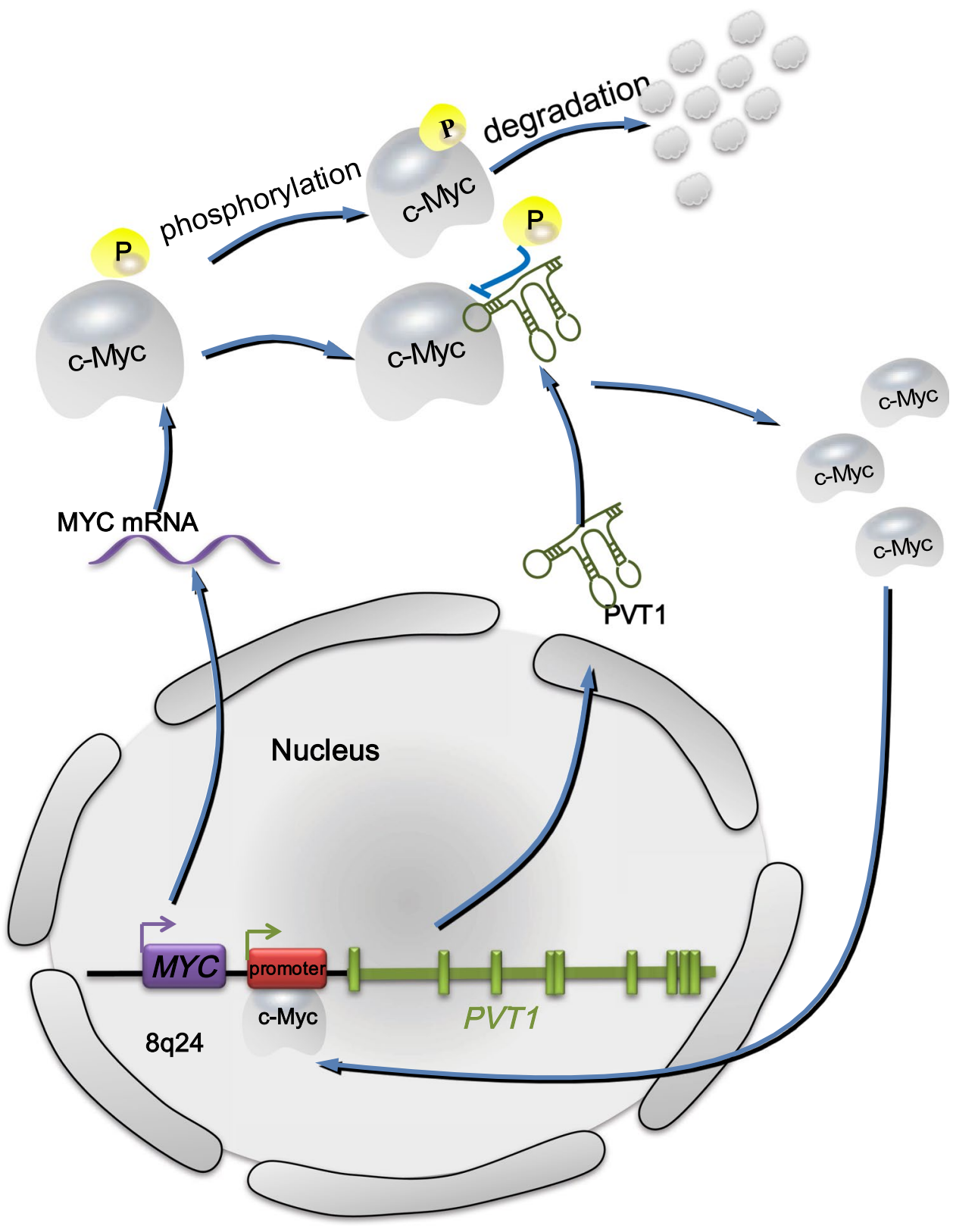

cancer-promoting effect" $[8,77]$. Moreover, they also found that $M Y C$ mRNA was significantly upregulated in cells after silencing the $P V T 1$ promoter and the role of $P V T 1$ promoter was independent of its IncRNA function, which affects the transcription level of $M Y C$. On chromosome 8, the distance between MYC and PVTl is $53 \mathrm{~kb}$, so how does the PVT1 promoter affect $M Y C$ transcription despite this relatively long distance barrier? Cho et al. used the Hi-ChIP method to target the enhancer-associated marker histone 3 lysine 27 acetylation. The promoter of $P V T 1$ and its four intragenic enhancers and the $M Y C$ promoter are in the same TAD of discrete self-interacting units of three-dimensionally organized chromatin [78]. Normally, the four intragenic enhancers of $P V T 1$ contact their own $P V T 1$ promoter. However, in cells in which the $P V T 1$ promoter is silent, the enhancers of $P V T 1$ can contact the $M Y C$ promoter more readily and reduce their association with the $P V T 1$ promoter. Silencing the enhancers of $P V T 1$ could reverse the upregulation of $M Y C$ induced by silencing of the PVT1 promoter. According to the previously described experimental results, a "promoter-enhancer competition" model was proposed (Fig. 3), which sheds light on the recurrent chromosomal rearrangements within the $M Y C-P V T 1$ locus.

The transcription of $P V T 1$ and $M Y C$ starts at the promoter core sequence for both genes, and RNA polymerase II (Pol II) is recruited by the transcription complex and accurately locates the specific region [79]. There is a promoter proximal pause mediated by a transcriptional pause-inducing factor 
Fig. 3 Promoter-enhancer competition between $P V T 1$ and $M Y C$. a When the $P V T 1$ promoter functions normally, the internal enhancer of $P V T 1$ (822E, 866E, 912E, 919E) tends to enhance the transcriptional efficiency of transcription factors starting from the $P V T 1$ promoter. The transcription depends on phosphorylation of the Ser 2 site of Pol II by BRD4 after phosphorylation of the Ser 5 site and P-TEFb is recruited by BRD4 to restart transcription. $\mathbf{b}$ In breast cancer and malignant lymphoma cells, the $P V T 1$ promoter sequence mutates, and the change in the topology enables the MYC promoter to interact with enhancer of $P V T 1$ frequently, upregulating the expression of $M Y C$ will be
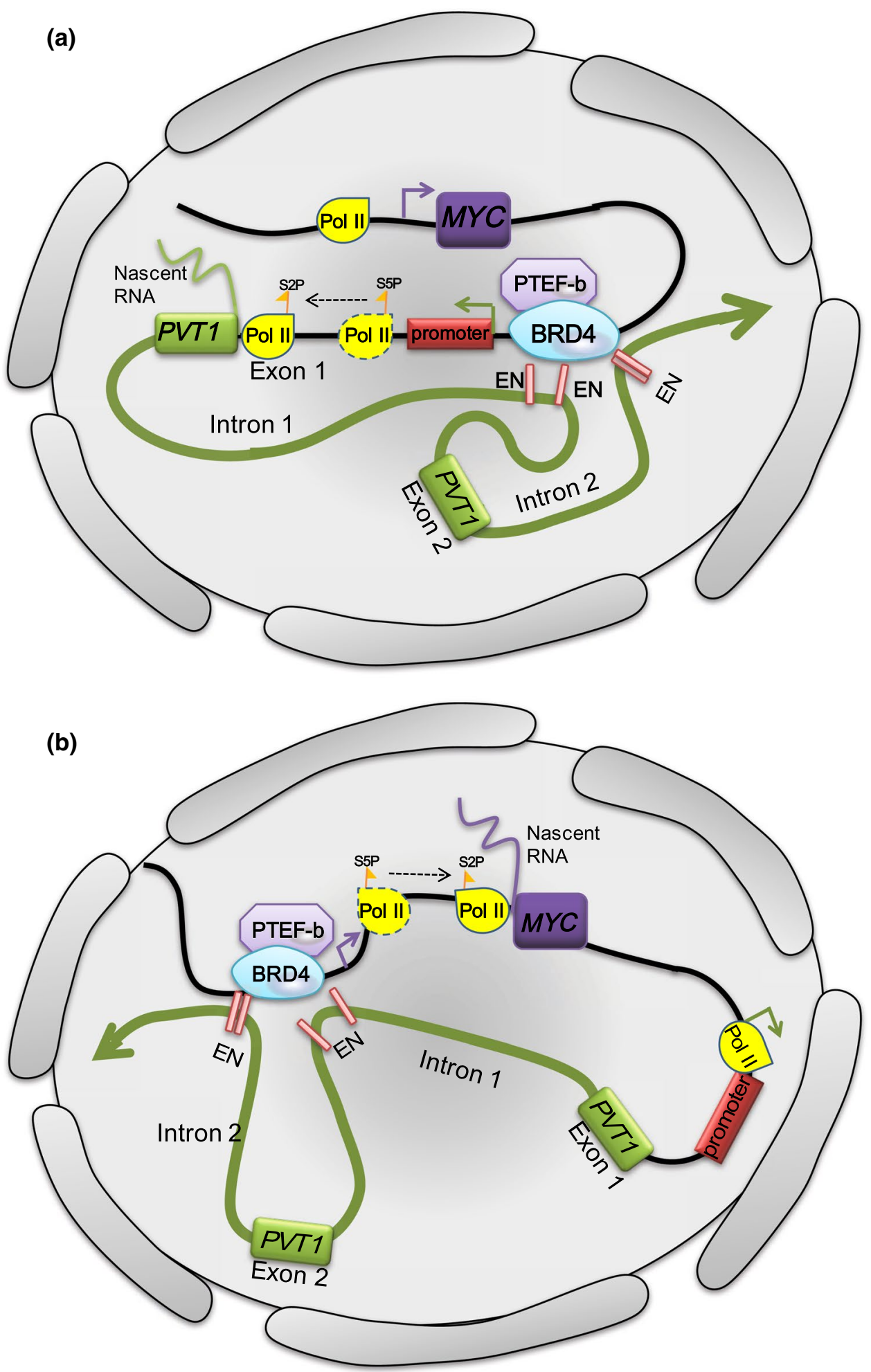

during transcriptional elongation in RNA synthesis, and the epigenetic reading protein bromodomain-containing protein 4 (BRD4) recruits P-TEFb, a kinase-positive transcription elongation factor, to the pretranscriptional initiation complex [80]. BRD4 can bind to the carboxy terminal domain of Pol II and phosphorylate it at the Ser 2 site, and P-TEFb dissociates the pause-inducing factor from the transcription complex to synergistically promote transcription via Pol II [81]. In normal tissues, the four internal enhancers of $P V T 1$ are preferentially contacted by the $P V T 1$ promoter; thus, BRD4 will preferentially occupy the $P V T 1$ promoter, reducing the occupation of BRD4 at the $M Y C$ promoter and decreasing the transcription of $M Y C$. 
Therefore, the $P V T 1$ promoter can act as a tumor suppressor DNA element of $M Y C$. In human breast cancer and malignant lymphoma, the $P V T 1$ promoter is mutated. Thus, the $M Y C$ promoter predominates the promoter-enhancer competition, thereby increasing the $M Y C$ mRNA level (Fig. 3). According to the description of the topology structure, the $P V T 1$ promoter, $M Y C$ promoter, and the four enhancers belong to the same TAD in normal tissues, while in cancer cells, the circumstance is different. Mutations in the PVTI promoter lead to changes in chromosome 3D construction so that the four intragenic enhancers of $P V T 1$ become closer to the MYC promoter and preferentially contact it. Thus, the promoter of $P V T 1$ inhibits the expression of its "neighbor" gene $M Y C$ by competing for the four enhancers on the same chromosome. We hypothesize that the "promoter-enhancer competition" model is the initial step in the $M Y C$-driven carcinogenic process. The high level of MYC transcripts is involved in the positive feedback mechanism previously described, activates the $P V T 1$ promoter and promotes PVT1 expression, which is consistent with the high expression of both MYC and PVT1 in tumor cells.

Additionally, in some tumor tissues, the region near the $P V T 1$ promoter shows more pronounced structural changes than other lncRNA promoters, such as deletions, inversions, or duplications, which alter the chromatin and TAD environment of the PVT1 promoter [9]. In human (ER-) HER2+ breast cancer, the $P V T 1$ intron 1, which is close to the $P V T 1$ promoter, is abnormally prone to cleavage, which might result in the fusion of PVTl with another gene on the same chromosome leading to an abnormal transcription process not regulated by the $P V T 1$ promoter.

In normal cells, studies have demonstrated low expression of PVT1 and MYC [72]. If the "promoter-enhancer competition" between PVTI and MYC commonly exists in cells, the PVTI promoter serves as a tumor suppressor, and will further downregulate the expression of $M Y C$, preventing the development of $M Y C$-driven tumors. Thus, the mutation of $P V T 1$ promoter will be the last straw in cancer development. However, the "promoter-enhancer competition" between $P V T 1$ and $M Y C$ is currently only observed in breast cancer cell lines and mutation of the $P V T 1$ promoter is only observed in breast cancer and malignant lymphoma. Thus, whether the competition mechanism acts as a complement and improves the positive feedback mechanism in common $M Y C$-driven tumors requires further analysis.

\section{PVT1 participates in the downstream signaling pathway of c-Myc by regulating key molecules}

In addition to preventing the phosphorylation of c-Myc to maintain its stability, PVT1 can participate in the downstream signaling pathway of c-Myc by regulating its downstream key factors (Fig. 4). PVT1 may promote the tumorigenesis and development of tumors together with c-Myc by regulating key molecules in the downstream signaling pathway of c-Myc. Therefore, we adequately understand that PVT1 and c-Myc jointly regulate key molecules, which may contribute to the development of new and selective anti-cancer drugs for the targeted therapy of cancers with high expression of PVT1 and c-Myc.

\section{PVT1 participates in the proliferation-related signaling pathway downstream of c-Myc}

Malignant cell proliferation is one of the hallmarks of tumor cells [82-86]. Research has shown that c-Myc can promote the transcription of positive cell cycle regulatory factors, such as E2F transcription factors, cyclin D1, and cyclin-dependent kinase 4 (CDK4). They can promote activation of the cell cycle and thus cell proliferation [87-90]. By inhibiting cell cycle inhibitors, such as p15, p16, and p21, which inhibit the cyclin-cdk complex, c-Myc promotes cell proliferation. In cancer cells, a complex of c-Myc and c-Myc-interacting zinc finger protein-1 (Miz1) act on the promoter of p15 and p16, which, in turn, downregulates the expression of $\mathrm{p} 15$ and $\mathrm{p} 16$, enabling the cell to enter the cell cycle phases. By binding to Miz1, c-Myc can also inhibit the expression of p21 [91]. C-Myc also inhibits the expression of p21 through miR-17, miR-20a, and miR-10b [92, 93]. Moreover, the transcription factor AP4 (TFAP4), induced by c-Myc, binds to the promoter of p21 and inhibits the expression of p21 [94, 95].

Recent studies showed that PVT1 affects the c-Mycrelated pathway and influences the cell cycle. PVT1 can promote tumor proliferation by downregulating the expression of p15 and p21 [96]. PVT1 acts on the promoter of $P 15$ and $P 16$ by binding the enhancer of zeste homolog 2 (EZH2) to inhibit p15 and p16 expression [97]. In cancer cells, knockdown of PVT1 expression inhibits the expression of cyclin D1 and CDK4, which suggests that PVT1 positively regulates the expression of cyclin D1 and CDK4 [98]. PVT1 inhibits the promoter of miR-200c by recruiting EZH2, which leads to an upregulation of cyclin D1 expression [99]. Furthermore, Chen et al. [22] confirmed that circPVT1 competently binds to the miR-125 family and enhances the expression of the transcription factor E2Fs. Therefore, PVT1 participates in the proliferationrelated signaling pathway downstream of c-Myc by regulating p21, p15/p16, CD4, and cyclin D (Fig. 4).

\section{PVT1 participates in the downstream angiogenesis-related signaling pathway of c-Myc}

Sustained angiogenesis is a key element in tumorigenesis. In an anoxic environment inside a tumor mass, hypoxia-inducible 


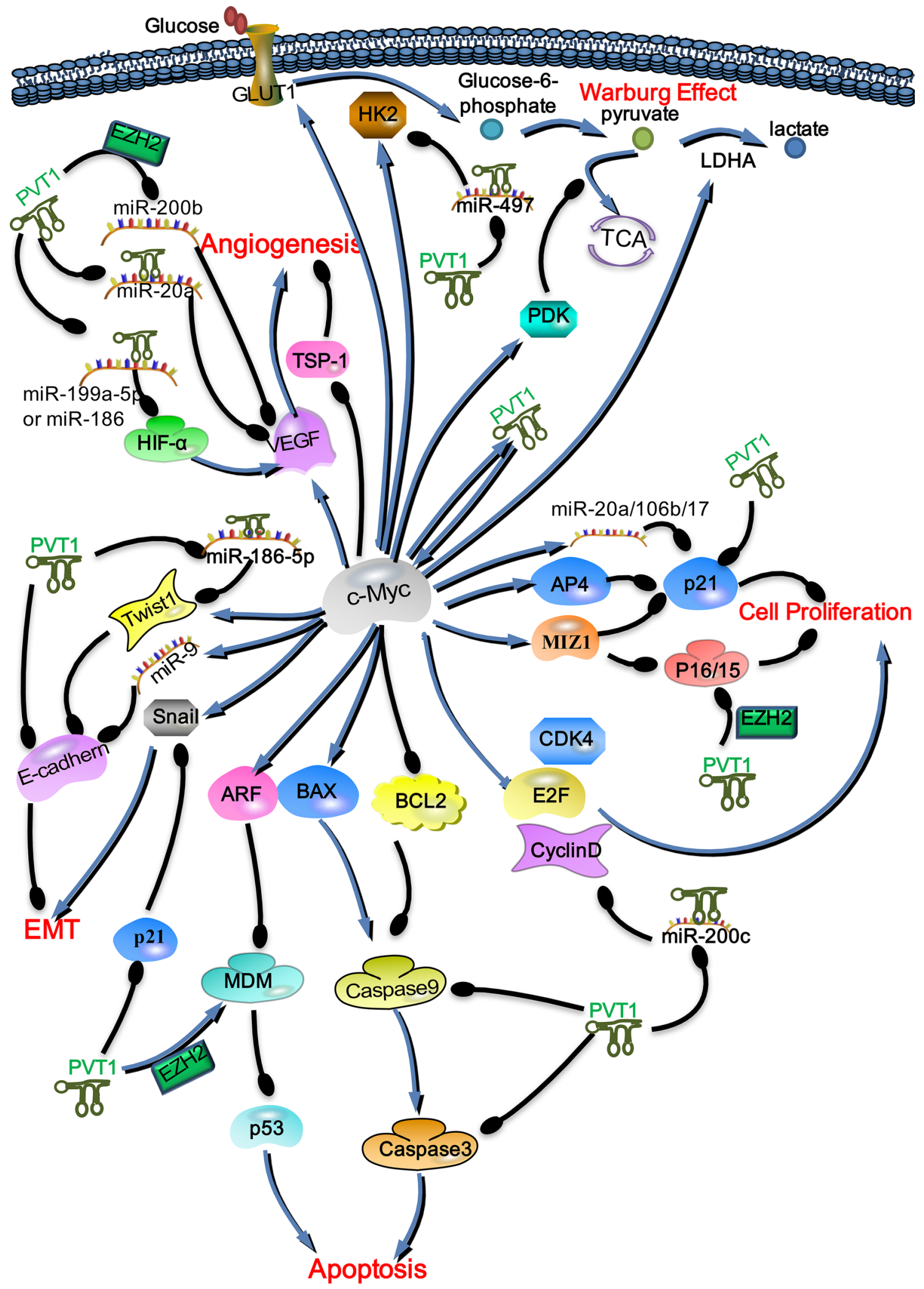


4Fig. 4 PVT1 participates in the downstream signaling pathway of c-Myc by regulating key molecules. In cancer cells, PVT1 not only exhibits a positive feedback interaction with c-Myc but also participates in the signal pathways downstream of c-Myc to promote the occurrence and development of tumors. PVT1 participates in the downstream signaling pathway of c-Myc by regulating the expression of key molecules. PVT1, similar to c-Myc, can promote tumor proliferation, angiogenesis, the EMT and the Warburg effect. Moreover, PVT1 may inhibit c-Myc-induced apoptosis by regulating caspase9, caspase3, and MDM. In this figure; the blue arrow indicates promotion/upregulation, while the black line indicates inhibition/downregulation

factor-1 (HIF-1) induces the expression of genes involved in angiogenesis [100-102]. HIF-1 $\alpha$ is a crucial oxygen sensor and plays a leading role in angiogenesis. By binding to the promoter of vascular endothelial growth factor (VEGF), HIF-1 $\alpha$ upregulates the expression of VEGF [103]. VEGF can act on the vascular endothelium, accelerating its proliferation and tube formation and increasing vessel permeability. In the angiogenesis signaling pathway, VEGF-A and thrombospondin-1 (TSP1) are angiogenesis inducers and inhibitors, respectively. TSP1 is a key balance factor in angiogenesis. It also binds to transmembrane receptors on endothelial cells, triggering inhibitory signals that counteract angiogenesis stimulation [104]. In cancer cells, c-Myc increases the transcriptional level of HIF-1 $\alpha$ [105], thus increasing the expression of HIF- $1 \alpha$, further promoting the expression of VEGF, which participates in downstream signaling pathways and promotes tumor angiogenesis. c-Myc can also directly upregulate the expression of VEGF [106] and inhibit the transcription of TSP1 [107], further promoting tumor angiogenesis. High expression of PVT1 in tumor cells can be used as a competing endogenous RNA sponge to adsorb miR-199a-5p or miR-186 and subsequently upregulate the expression of HIF- $1 \alpha$ [108, 109]. PVT1 can recruit EZH2 to the $m i R-200 b$ promoter and inhibit miR-200b expression [110]. MiR-200b reduces the VEGF level [111]. Thus, PVT1 may upregulate the expression level of VEGF through inhibiting miR-200b. Moreover, PVT1 may increase the expression of VEGF by inhibiting miR-20a [13, 112]. Therefore, PVT1 participates in the downstream angiogenesisrelated signaling pathway of c-Myc and can promote tumor angiogenesis (Fig. 4).

\section{PVT1 participates in the downstream epithelial- mesenchymal transition-related signaling pathway of c-Myc}

Metastasis of tumors typically leads to patient death [113-117]. Studies have shown that the high expression and amplification of the $M Y C$ gene can promote tumor metastasis, which is closely related to the epithelial-mesenchymal transition (EMT) of tumor cells [118, 119]. The mesenchymal state is characterized by high expression of vimentin and low expression of E-cadherin. Decreased levels of
E-cadherin can result in reduced adhesion of cells, which enables them to acquire characteristics that make them prone to invasion and metastasis. The loss of E-cadherin expression and upregulation of snail homolog 1 (Snail1), Twist1, and a number of other proteins are considered important features of the EMT. In tumor cells, the TGF- $\beta$ signaling pathway induces Snail, which further inhibits the expression of E-cadherin, resulting in a loss of adhesion between cells. After knocking out $M Y C$, the expression of Snail induced by the TGF- $\beta$ signaling pathway decreased because c-Myc promoted the transcription of Snail and stabilized it [119, 120]. Studies have shown that c-Myc can induce the production of miR-9, which promotes the migration and invasion of cancer cells by inhibiting the cell adhesion protein E-cadherin [118, 121, 122]. Twist1 has been shown to promote the EMT by directly inhibiting epithelial markers, such as E-cadherin, and upregulating mesenchymal markers, such as N-cadherin [123]. Twist1 is the downstream transcription target of c-Myc, and c-Myc can directly enhance the transcription of Twist1 [124].

Studies have shown that elevated expression of PVT1 can induce a decrease in E-cadherin [125, 126]. Inhibition of the expression of PVT1 in cancer cells reduces the expression of vimentin, while it enhances the expression of E-cadherin [98]. PVT1 downregulates the expression of p21, while $\mathrm{p} 21$ downregulates the expression of Snail [127]. Moreover, PVT1 can upregulate Twist1 by adsorption of miR-186-5p to promote the EMT [128]. Therefore, PVT1 participates in the proliferation-related signaling pathway downstream of c-Myc by affecting Snail1, Twist1, and E-cadherin (Fig. 4).

\section{PVT1 participates in the downstream Warburg effect-related signaling pathway of c-Myc}

Cancer is often accompanied by deregulating cellular energetic [129-133]. The pyruvate produced by glycolysis does not enter the mitochondria and is converted to lactic acid by lactate dehydrogenase (LDH), which typically occurs when normal cells are in a low-oxygen state. However, in cancer cells, the production of lactic acid under aerobic conditions is referred to as "aerobic glycolysis" or the Warburg effect. Many genes involved in glucose metabolism have been reported to be directly upregulated by the induction of c-Myc, particularly glucose transporter 1 (GLUT1) and hexokinase 2 (HK2) [134]. C-Myc also regulates pyruvate dehydrogenase kinase (PDK), which blocks pyruvate from entering the TCA cycle. C-Myc activates lactate dehydrogenase $\mathrm{A}$, which converts pyruvate to lactic acid. PVT1 acts as an endogenous competitive RNA to inhibit miR-497 whose downstream target is $\mathrm{HK} 2$, which results in elevated HK2 expression, increasing glucose consumption and glycolysis [135]. Therefore, PVT1 can regulate HK2 expression to 
participate in the downstream Warburg effect-related signaling pathway of c-Myc (Fig. 4).

\section{PVT1 participates in the downstream apoptosis-related signaling pathway of c-Myc}

In most human cancers, c-Myc expression is deregulated and/or significantly increased. Interestingly, high levels of c-Myc overexpression not only induce ARF transcription but also stabilize ARF by inhibiting ubiquitin ligase, which, in turn, leads to p53 activation, induces apoptosis, and limits c-Myc-induced carcinogenesis [136]. Mouse double minute 2 homolog (MDM2) downregulates p53 by binding to and inhibiting the transactivation domain of p53 and promoting its degradation [137]. However, ARF can promote the degradation of MDM2, which, in turn, leads to p53 activation. Inactivation of the Arf-Mdm2-p53 tumor suppressor pathway is believed to be a crucial step in tumorigenesis [138]. C-Myc induces apoptosis by regulating the ARFMDM2-p53 pathway to limit its own carcinogenic potential [136]. However, PVT1 can increase the stability of EZH2 protein by binding to EZH2, which promotes the expression of MDM2 protein. In cancer cells, high expression of PVT1 enhances the expression of EZH2 and MDM2, inhibits the expression of P53 protein in cancer cells, and thus exerts anti-apoptotic effects [139, 140]. C-Myc can also inhibit anti-apoptotic proteins, such as BCL-2 and BCL-XL, leading to a certain amount of tumor apoptosis [141]. When c-Myc exerts a pro-apoptotic function, it can further activate caspase- 9 and caspase- 3 to induce apoptosis by increasing the expression of and activating the pro-apoptotic protein Bax [142]. PVT1 can downregulate the expression of caspase- 9 , caspase-7, and poly ADP-ribose polymerase, thereby inhibiting apoptosis and ultimately leading to radiation tolerance [14]. Studies have also shown that PVT1 can downregulate the expression of caspase-3 to inhibit apoptosis [143, 144]. Therefore, PVT1 may participates in the downstream apoptosis-related signaling pathway of c-Myc by affecting MDM, caspase-9, and caspase-3 (Fig. 4).

\section{Perspectives}

In addition to the interaction between PVT1 and MYC, the newly discovered molecule CircPVT1 is also associated with MYC [145-149]. CircPVT1 is highly expressed in cancer and can mediate the expression of c-Myc [22, 150]. Let-7 can target $M Y C$ mRNA and downregulate its expression; however, circPVT1 can impede let-7 through its sponge adsorption effect and increase c-Myc expression [22,151]. In acute lymphocytic leukemia, circPVT1 can upregulate the expression of c-Myc and anti-apoptotic Bcl-2 proteins [23]. After deletion of circPVT1, the expression levels of c-Myc and $\mathrm{Bcl}-2$ proteins were significantly reduced, while the level of PVT1 RNA was not changed [23]. However, CircPVT1 has many other mechanisms that have not been elucidated. It is worth further investigating whether CircPVT1 has similar mechanisms to PVT1 in cancer cells, whether CircPVT1 interacts with MYC, and which molecules CircPVT1 can regulate to participate in the downstream signaling pathway of c-Myc. Moreover, there are no studies on PVT1-encoded microRNAs that indicate whether they interact with MYC (including miR-1204, miR-1205, miR-1206, miR-1207-5p, miR-1207-3p, and miR-1208) [20, 21].

Currently, the mechanism of action of PVT1 in cancer and the interaction mechanism with MYC are not completely clear; however, in-depth studies of PVT1 can contribute to the development of new therapeutic targets. C-Myc is an important protein in cells and participates in many important metabolic pathways. Overexpression of c-Myc in cancer substantially enhances certain metabolic pathways. If c-Myc is directly inhibited, therapeutic interventions will have a strong impact on patients [152]. Therefore, utilizing a small molecular drug to inhibit PVT1, compared to treatment that directly targets MYC, would fine-tune the level of c-Myc in cancer and reduce toxic side effects or exert an influence on the c-Myc downstream biological phenotype, which is another therapeutic target in $M Y C$-driven cancer. Recently, antisense LNA GapmeRs have been used by researchers to degrade PVT1 in acute erythroleukemia cell lines, which can increase apoptosis and necrosis of tumor cells. They suggested that PVT1 antisense LNA GapmeRs can be used alone or combined with chemotherapeutic drugs in the treatment of acute erythroleukemia [153]. PVT1 promotes the development of cisplatin resistance in colorectal cancer; thus, silencing PVT1 inhibits tumorigenesis and cisplatin resistance in colorectal cancer [154]. Knockdown of PVT1 to a certain extent enhances the radiosensitivity of non-small cell lung cancer cells through inhibiting cell proliferation and promoting apoptosis, which provides a new therapeutic target for improving the efficiency of radiotherapy in patients with non-small cell lung cancer [155]. In addition, studies have found that gemcitabine can inhibit the growth of pancreatic cancer cells by decreasing PVT1 levels and increasing PVT1 encoded miRNAs, such as the miR-1207 pair (miR-1207-5p/3p) [156, 157]. Therefore, there is a bright future for research on how to use PVT1 as a therapeutic target. It is necessary to develop drugs that target PVT1. In the future, we can interfere with the function of PVT1 in tumor cells using siRNAs or antisense LNA GapmeRs against PVT1. Further in vivo studies and clinical trials are required to evaluate the feasibility of this strategy. Moreover, according to the latest promoter competition mechanism, the $P V T 1$ promoter, as a "non-standard" tumor suppressor, is a novel candidate for designing a new therapeutic strategy for tumors in which $M Y C$ serves as a carcinoma driver. 
Furthermore, the high and specific expression of PVT1 in human cancer indicates its potential as a biomarker in early clinical diagnosis. PVT1 is considered an indicator of poor prognosis in cancer because PVT1 has been shown to play a role in cell proliferation, cell apoptosis, and cell migration, which are key elements of poor prognosis in human cancer $[13,128]$. The expression of PVT1 is upregulated in gastric cancer tissues and significantly associated with advanced tumor and lymph node metastasis. Upregulated PVT1 in gastric cancer can promote the proliferation and invasion of gastric cancer cells, which is associated with poor prognosis [97, 108, 158]. A high PVT1 expression level in patients with prostate cancer has been shown to be associated with low overall survival. PVT1 expression is significantly correlated with tumor stage and can promote tumor cell proliferation, invasion, and metastasis in prostate cancer [128, 144]. The expression of PVT1 in ovarian cancer tissues is higher than that in normal ovarian tissue and is related to the advanced stage of ovarian cancer and lower overall survival. The high expression of PVT1 in ovarian cancer cells promotes the proliferation, migration, and invasion ability of ovarian cancer cells $[159,160]$. In gliomas, PVT1 is also highly expressed. PVT1 can promote the development of glioma cells through various mechanisms, resulting in a worse prognosis for patients with glioma and high PVT1 expression [18, 161, 162]. Multiple meta-analyses have also shown that PVT1 can be used as a new tumor biomarker and a predictor of poor prognosis in different cancers [163-167].

\section{Conclusions}

We have summarized the latest research regarding the relationship between PVT1 and MYC. At the chromosomal level, PVT1 is easily fused with other genes to form a fusion gene that drives the abnormal expression of cancerassociated proteins. Moreover, GWAS also indicated that $P V T 1$ and MYC located in the 8q24 segment are associated with cancer risk. Furthermore, there is a synergistic effect of positive feedback between MYC and PVT1, which results in increased expression levels of these genes in cancer. With the development of 3D technology, the latest research shows that PVT1 and MYC have a "promoter-enhancer competition mechanism" in a three-dimensional structure. Moreover, PVT1 can also participate in the c-Myc downstream signaling pathway by acting on key molecules downstream of c-Myc.

However, the interaction between PVT1 and MYC has not been fully understood, and further studies are needed. Studies have shown that PVT1 can inhibit the phosphorylation and degradation of c-Myc to promote its stability; however, are there ways that PVT1 impacts the transcription and translation of c-Myc? In addition to gene fusion, the positive feedback mechanism, coamplification and the "promoterenhancer competition mechanism", are there other mechanisms of that mediate the PVT1 and MYC interaction? The "promoter-enhancer competition" between PVT1 and MYC is currently only found in breast cancer cell lines and, thus, requires further exploration regarding whether this competition commonly exists in other $M Y C$-driven cancers. Moreover, the key molecules that are at the c-Myc downstream signaling pathway and regulated by PVT1 are needed to further verify and clarify to develop anticancer drugs by inhibiting these key molecules. In addition, it is worth elaborating on whether microRNAs and the circPVT1 encoded by $P V T 1$ interact with MYC.

Although the increasing number of mechanisms of action for PVT1 and its interaction with MYC in cancer are being discovered, there is no application for PVT1 in clinical treatment to date, and only one clinical trial on PVT1 is recruiting subjects. Thus, the findings resulting from such studies are expected to be transformed into methods of early cancer diagnosis and treatment applications in the future.

Author contributions KJ, SW, YZ, MX, YM gathered the related literature and drafted the manuscript. XL, GL, WX, ZZ, YH participated in the design of the review and drafted the manuscript. All authors read and approved the final manuscript.

Funding This work was supported in part by Grants from the National Natural Science Foundation of China (81572787, 81672683, 81672993, 81772928, and 81872278), the Overseas Expertise Introduction Project for Discipline Innovation (111 Project, No. 111-2-12), and the Natural Science Foundation of Hunan Province (2016JC2035, 2017SK2105, 2018JJ3704, 2018JJ3815, 2018SK21210, 2018SK21211 and 2019JJ50354)

\section{Compliance with ethical stsndards}

Conflict of interest The authors declare that they have no competing interests.

Open Access This article is distributed under the terms of the Creative Commons Attribution 4.0 International License (http://creativeco mmons.org/licenses/by/4.0/), which permits unrestricted use, distribution, and reproduction in any medium, provided you give appropriate credit to the original author(s) and the source, provide a link to the Creative Commons license, and indicate if changes were made.

\section{References}

1. Parolia A, Cieslik M, Chinnaiyan AM (2018) Competing for enhancers: PVT1 fine-tunes MYC expression. Cell Res 28(8):785-786

2. Fan C et al (2019) Long non-coding RNA LOC284454 promotes migration and invasion of nasopharyngeal carcinoma via modulating the Rho/Rac signaling pathway. Carcinogenesis 40(2):380-391 
3. Lian Y et al (2018) Long noncoding RNA AFAP1-AS1 acts as a competing endogenous RNA of miR-423-5p to facilitate nasopharyngeal carcinoma metastasis through regulating the Rho/Rac pathway. J Exp Clin Cancer Res 37(1):253

4. Wei F et al (2019) Cloning and characterization of the putative AFAP1-AS1 promoter region. J Cancer 10(5):1145-1153

5. Bo $\mathrm{H}$ et al (2018) High expression of lncRNA AFAP1-AS1 promotes the progression of colon cancer and predicts poor prognosis. J Cancer 9(24):4677-4683

6. Bo $\mathrm{H}$ et al (2019) Upregulation and hypomethylation of lncRNA AFAP1AS1 predicts a poor prognosis and promotes the migration and invasion of cervical cancer. Oncol Rep 41(4):2431-2439

7. Cui M et al (2016) Long non-coding RNA PVT1 and cancer. Biochem Biophys Res Commun 471(1):10-14

8. Colombo T et al (2015) PVT1: a rising star among oncogenic long noncoding RNAs. Biomed Res Int 2015:304208

9. Cho SW et al (2018) Promoter of lncRNA gene PVT1 is a tumorsuppressor DNA boundary element. Cell 173(6):1398-1412.e22

10. Murugan AK, Munirajan AK, Alzahrani AS (2018) Long noncoding RNAs: emerging players in thyroid cancer pathogenesis. Endocr Relat Cancer 25(2):R59-R82

11. Zhao J et al (2018) LncRNA PVT1 promotes angiogenesis via activating the STAT3/VEGFA axis in gastric cancer. Oncogene 37(30):4094-4109

12. Ding $\mathrm{H}$ et al (2018) Long noncoding RNA PVT1 inhibits interferon-alpha mediated therapy for hepatocellular carcinoma cells by interacting with signal transducer and activator of transcription 1. Biochem Biophys Res Commun 500(4):973-980

13. Huang F et al (2018) LncRNA PVT1 triggers cyto-protective autophagy and promotes pancreatic ductal adenocarcinoma development via the miR-20a-5p/ULK1 Axis. Mol Cancer 17(1):98

14. He Y et al (2018) Long non-coding RNA PVT1 predicts poor prognosis and induces radioresistance by regulating DNA repair and cell apoptosis in nasopharyngeal carcinoma. Cell Death Dis 9(2):235

15. Martini P et al (2017) lncRNAs as novel indicators of patients' prognosis in stage I epithelial ovarian cancer: a retrospective and multicentric study. Clin Cancer Res 23(9):2356-2366

16. Zhao L et al (2018) LncRNA-PVT1 promotes pancreatic cancer cells proliferation and migration through acting as a molecular sponge to regulate miR-448. J Cell Physiol 233(5):4044-4055

17. Wang W et al. (2019) PVT1 promotes cancer progression via microRNAs. Front Oncol 9:609

18. Fu C et al (2018) LncRNA PVT1 facilitates tumorigenesis and progression of glioma via regulation of MiR-128-3p/ GREM1 axis and BMP signaling pathway. Neurotherapeutics 15(4):1139-1157

19. Zhang $Y$ et al (2018) A preliminary investigation of PVT1 on the effect and mechanisms of hepatocellular carcinoma: evidence from clinical data, a meta-analysis of 840 cases, and in vivo validation. Cell Physiol Biochem 47(6):2216-2232

20. Huppi $\mathrm{K}$ et al (2008) The identification of microRNAs in a genomically unstable region of human chromosome 8q24. Mol Cancer Res 6(2):212-221

21. Beck-Engeser GB et al (2008) Pvt1-encoded microRNAs in oncogenesis. Retrovirology 5:4

22. Chen J et al (2017) Circular RNA profile identifies circPVT1 as a proliferative factor and prognostic marker in gastric cancer. Cancer Lett 388:208-219

23. Hu J et al (2018) Circular RNA PVT1 expression and its roles in acute lymphoblastic leukemia. Epigenomics 10(6):723-732

24. Tang J et al (2018) LncRNA PVT1 regulates triple-negative breast cancer through KLF5/beta-catenin signaling. Oncogene 37(34):4723-4734
25. Riedell PA, Smith SM (2018) Double hit and double expressors in lymphoma: definition and treatment. Cancer 124(24):4622-4632

26. Stine ZE et al (2015) MYC, metabolism, and cancer. Cancer Discov 5(10):1024-1039

27. Caforio M et al (2018) Recent advances in searching c-Myc transcriptional cofactors during tumorigenesis. J Exp Clin Cancer Res 37(1):239

28. Tu C et al (2018) Identification of genomic alterations in nasopharyngeal carcinoma and nasopharyngeal carcinoma-derived Epstein-Barr virus by whole-genome sequencing. Carcinogenesis 39(12):1517-1528

29. Tong $\mathrm{Y}$ et al (2018) Cumulative evidence for relationships between multiple variants in 8q24 and colorectal cancer incidence. Medicine (Baltimore) 97(35):e11990

30. Shi J et al (2016) Fine-scale mapping of 8q24 locus identifies multiple independent risk variants for breast cancer. Int J Cancer 139(6):1303-1317

31. Han J et al (2017) Genetic variants within the cancer susceptibility region 8q24 and ovarian cancer risk in Han Chinese women. Oncotarget 8(22):36462-36468

32. Shen $C$ et al (2017) Long-distance interaction of the integrated HPV fragment with MYC gene and 8q24.22 region upregulating the allele-specific MYC expression in HeLa cells. Int J Cancer 141(3):540-548

33. Schwartz M, Zlotorynski E, Kerem B (2006) The molecular basis of common and rare fragile sites. Cancer Lett 232(1):13-26

34. Ferber MJ et al (2004) Positioning of cervical carcinoma and Burkitt lymphoma translocation breakpoints with respect to the human papillomavirus integration cluster in FRA8C at 8q24.13. Cancer Genet Cytogenet 154(1):1-9

35. Toledo F et al (1992) Co-amplified markers alternate in megabase long chromosomal inverted repeats and cluster independently in interphase nuclei at early steps of mammalian gene amplification. EMBO J 11(7):2665-2673

36. Hellman A et al (2002) A role for common fragile site induction in amplification of human oncogenes. Cancer Cell 1(1):89-97

37. L'Abbate A et al (2014) Genomic organization and evolution of double minutes/homogeneously staining regions with MYC amplification in human cancer. Nucleic Acids Res 42(14):9131-9145

38. Kim D et al (2016) A small molecule inhibits Akt through direct binding to Akt and preventing Akt membrane translocation. J Biol Chem 291(43):22856

39. Northcott PA et al (2012) Subgroup-specific structural variation across 1,000 medulloblastoma genomes. Nature 488(7409):49-56

40. Tseng YY, Bagchi A (2015) The PVT1-MYC duet in cancer. Mol Cell Oncol 2(2):e974467

41. Sarver AL et al (2016) MYC and PVT1 synergize to regulate RSPO1 levels in breast cancer. Cell Cycle 15(7):881-885

42. Li C et al (2018) Identification of RSPO2 fusion mutations and target therapy using a porcupine inhibitor. Sci Rep 8(1):14244

43. Shen $P$ et al (2018) Comprehensive genomic profiling of neuroendocrine bladder cancer pinpoints molecular origin and potential therapeutics. Oncogene 37(22):3039-3044

44. Ĺabbate A et al (2018) MYC-containing amplicons in acute myeloid leukemia: genomic structures, evolution, and transcriptional consequences. Leukemia 32(10):2152-2166

45. Taniwaki M (2015) Recent advancements in molecular cytogenetics for hematological malignancies: identification of novel PVT1 fusion genes. Rinsho Ketsueki 56(10):2056-2065

46. Chinen $Y$ et al (2014) 8q24 amplified segments involve novel fusion genes between NSMCE2 and long noncoding RNAs in acute myelogenous leukemia. J Hematol Oncol 7:68

47. Okuda T et al (2017) Molecular heterogeneity in the novel fusion gene APIP-FGFR2: diversity of genomic breakpoints in gastric 
cancer with high-level amplifications at $11 \mathrm{p} 13$ and 10q26. Oncol Lett 13(1):215-221

48. Nakamura $\mathrm{Y}$ et al (2015) Identification of SUPT3H as a novel 8q24/MYC partner in blastic plasmacytoid dendritic cell neoplasm with $\mathrm{t}(6 ; 8)(\mathrm{p} 21 ; \mathrm{q} 24)$ translocation. Blood Cancer J 5:e301

49. Shtivelman E, Bishop JM (1990) Effects of translocations on transcription from PVT. Mol Cell Biol 10(4):1835-1839

50. Xu PX et al (1997) Mouse Eya genes are expressed during limb tendon development and encode a transcriptional activation function. Proc Natl Acad Sci USA 94(22):11974-11979

51. Pleasance ED et al (2010) A small-cell lung cancer genome with complex signatures of tobacco exposure. Nature 463(7278):184-190

52. Zanke BW et al (2007) Genome-wide association scan identifies a colorectal cancer susceptibility locus on chromosome 8q24. Nat Genet 39(8):989-994

53. Haiman CA et al (2007) Multiple regions within 8q24 independently affect risk for prostate cancer. Nat Genet 39(5):638-644

54. Easton DF et al (2007) Genome-wide association study identifies novel breast cancer susceptibility loci. Nature 447(7148): 1087-1093

55. Ghoussaini M et al (2008) Multiple loci with different cancer specificities within the $8 \mathrm{q} 24$ gene desert. J Natl Cancer Inst 100(13):962-966

56. Wolpin BM et al (2014) Genome-wide association study identifies multiple susceptibility loci for pancreatic cancer. Nat Genet 46(9):994-1000

57. Obazee $\mathrm{O}$ et al (2018) Common genetic variants associated with pancreatic adenocarcinoma may also modify risk of pancreatic neuroendocrine neoplasms. Carcinogenesis 39(3):360-367

58. Moschovis D et al (2019) Association between genetic polymorphisms in long non-coding RNAs and pancreatic cancer risk. Cancer Biomark 24(1):117-123

59. Pharoah PD et al (2013) GWAS meta-analysis and replication identifies three new susceptibility loci for ovarian cancer. Nat Genet 45(4):362-370 (370e1-2)

60. Miele A, Dekker J (2008) Long-range chromosomal interactions and gene regulation. Mol BioSyst 4(11):1046-1057

61. Pomerantz MM et al (2009) The $8 \mathrm{q} 24$ cancer risk variant rs6983267 shows long-range interaction with MYC in colorectal cancer. Nat Genet 41(8):882-884

62. Pomerantz MM et al (2009) Evaluation of the 8q24 prostate cancer risk locus and MYC expression. Cancer Res 69(13):5568-5574

63. Meyer KB et al (2011) A functional variant at a prostate cancer predisposition locus at 8q24 is associated with PVT1 expression. PLoS Genet 7(7):e1002165

64. Ahmadiyeh $\mathrm{N}$ et al (2010) 8q24 prostate, breast, and colon cancer risk loci show tissue-specific long-range interaction with MYC. Proc Natl Acad Sci USA 107(21):9742-9746

65. Gong L et al (2018) Transcriptome-wide association study identifies multiple genes and pathways associated with pancreatic cancer. Cancer Med 7(11):5727-5732

66. Riquelme E et al (2014) Frequent coamplification and cooperation between C-MYC and PVT1 oncogenes promote malignant pleural mesothelioma. J Thorac Oncol 9(7):998-1007

67. Shtivelman E, Bishop JM (1989) The PVT gene frequently amplifies with MYC in tumor cells. Mol Cell Biol 9(3):1148-1154

68. Rao PH et al (2015) Coamplification of Myc/Pvt1 and homozygous deletion of Nlrp1 locus are frequent genetics changes in mouse osteosarcoma. Genes Chromosomes Cancer 54(12):796-808

69. Yeh E et al (2004) A signalling pathway controlling c-Myc degradation that impacts oncogenic transformation of human cells. Nat Cell Biol 6(4):308-318
70. Lu D et al (2017) lncRNA PVT1 in cancer: a review and metaanalysis. Clin Chim Acta 474:1-7

71. Johnsson P, Morris KV (2014) Expanding the functional role of long noncoding RNAs. Cell Res 24(11):1284-1285

72. Carramusa L et al (2007) The PVT-1 oncogene is a Myc protein target that is overexpressed in transformed cells. J Cell Physiol 213(2):511-518

73. van Arensbergen J, van Steensel B, Bussemaker HJ (2014) In search of the determinants of enhancer-promoter interaction specificity. Trends Cell Biol 24(11):695-702

74. Catarino RR, Stark A (2018) Assessing sufficiency and necessity of enhancer activities for gene expression and the mechanisms of transcription activation. Genes Dev 32(3-4):202-223

75. Fan C et al (2019) GPC6 promotes cell proliferation, migration, and invasion in nasopharyngeal carcinoma. J Cancer 10(17): 3926-3932

76. Tang $\mathrm{Z}$ et al (2015) CTCF-mediated human 3D genome architecture reveals chromatin topology for transcription. Cell 163(7):1611-1627

77. Tseng YY et al (2014) PVT1 dependence in cancer with MYC copy-number increase. Nature 512(7512):82-86

78. Marchese FP, Huarte M (2018) A "counter-enhancer" in tumor suppression. Cell 173(6):1318-1319

79. Lu X et al (2016) Multiple P-TEFbs cooperatively regulate the release of promoter-proximally paused RNA polymerase II. Nucleic Acids Res 44(14):6853-6867

80. Merkenschlager M, Odom DT (2013) CTCF and cohesin: linking gene regulatory elements with their targets. Cell 152(6):1285-1297

81. Yang Z et al (2005) Recruitment of P-TEFb for stimulation of transcriptional elongation by the bromodomain protein Brd4. Mol Cell 19(4):535-545

82. Xiao L et al (2019) TSC22D2 identified as a candidate susceptibility gene of multi-cancer pedigree using genome-wide linkage analysis and whole exome sequencing. Carcinogenesis. https:// doi.org/10.1093/carcin/bgz095

83. Zhang Y et al (2018) Function of the c-Met receptor tyrosine kinase in carcinogenesis and associated therapeutic opportunities. Mol Cancer 17(1):45

84. Wei F et al (2018) BPIFB1 (LPLUNC1) inhibits radioresistance in nasopharyngeal carcinoma by inhibiting VTN expression. Cell Death Dis 9(4):432

85. Xia $\mathrm{M}$ et al (2019) Communication between mitochondria and other organelles: a brand-new perspective on mitochondria in cancer. Cell Biosci 9:27

86. Xiong $\mathrm{F}$ et al (2019) Effects and mechanisms of innate immune molecules on inhibiting nasopharyngeal carcinoma. Chin Med J (Engl) 132(6):749-752

87. Keller UB et al (2007) Myc targets Cks 1 to provoke the suppression of p27Kip1, proliferation and lymphomagenesis. EMBO J 26(10):2562-2574

88. Mo Y et al (2019) Proteomic analysis of the molecular mechanism of lovastatin inhibiting the growth of nasopharyngeal carcinoma Cells. J Cancer 10(10):2342-2349

89. Yu Q, Ciemerych MA, Sicinski P (2005) Ras and Myc can drive oncogenic cell proliferation through individual D-cyclins. Oncogene 24(47):7114-7119

90. Hermeking $\mathrm{H}$ et al (2000) Identification of CDK4 as a target of c-MYC. Proc Natl Acad Sci USA 97(5):2229-2234

91. Wu Y et al (2019) Herpesvirus acts with the cytoskeleton and promotes cancer progression. J Cancer 10(10):2185-2193

92. Petrocca $\mathrm{F}$ et al (2008) E2F1-regulated microRNAs impair TGFbeta-dependent cell-cycle arrest and apoptosis in gastric cancer. Cancer Cell 13(3):272-286 
93. Fontana L et al (2008) Antagomir-17-5p abolishes the growth of therapy-resistant neuroblastoma through p21 and BIM. PLoS One 3(5):e2236

94. Wong PP et al (2012) Histone demethylase KDM5B collaborates with TFAP2C and Myc to repress the cell cycle inhibitor p21(cip) (CDKN1A). Mol Cell Biol 32(9):1633-1644

95. Jung P, Hermeking H (2009) The c-MYC-AP4-p21 cascade. Cell Cycle 8(7):982-989

96. Cui D et al (2016) Long non-coding RNA PVT1 as a novel biomarker for diagnosis and prognosis of non-small cell lung cancer. Tumour Biol 37(3):4127-4134

97. Kong R et al (2015) Long noncoding RNA PVT1 indicates a poor prognosis of gastric cancer and promotes cell proliferation through epigenetically regulating p15 and p16. Mol Cancer 14:82

98. Wang C et al (2018) Upregulated plasmacytoma variant translocation 1 promotes cell proliferation, invasion and metastasis in colorectal cancer. Mol Med Rep 17(5):6598-6604

99. Chen L et al (2018) Effect of long non-coding RNA PVT1 on cell proliferation and migration in melanoma. Int $\mathrm{J}$ Mol Med 41(3):1275-1282

100. Wang YA et al (2018) Effects of tumor metabolic microenvironment on regulatory T cells. Mol Cancer 17(1):168

101. Fan $\mathrm{C}$ et al (2018) The emerging role of Epstein-Barr virus encoded microRNAs in nasopharyngeal carcinoma. J Cancer 9(16):2852-2864

102. Tang L et al (2018) Role of metabolism in cancer cell radioresistance and radiosensitization methods. J Exp Clin Cancer Res 37(1):87

103. Wang X, Wang G, Wang Y (2009) Intravitreous vascular endothelial growth factor and hypoxia-inducible factor 1a in patients with proliferative diabetic retinopathy. Am J Ophthalmol 148(6):883-889

104. Kazerounian S, Yee KO, Lawler J (2008) Thrombospondins in cancer. Cell Mol Life Sci 65(5):700-712

105. Weili $Z$ et al (2019) Knockdown of USP28 enhances the radiosensitivity of esophageal cancer cells via the c-Myc/hypoxia-inducible factor-1 alpha pathway. J Cell Biochem 120(1):201-212

106. von Rahden BH et al (2006) c-myc amplification is frequent in esophageal adenocarcinoma and correlated with the upregulation of VEGF-A expression. Neoplasia 8(9):702-707

107. Watnick RS et al (2003) Ras modulates Myc activity to repress thrombospondin-1 expression and increase tumor angiogenesis. Cancer Cell 3(3):219-231

108. Huang $T$ et al (2017) The long noncoding RNA PVT1 functions as a competing endogenous RNA by sponging miR-186 in gastric cancer. Biomed Pharmacother 88:302-308

109. Wang C et al (2018) LncRNA PVT1 regulate expression of HIF1alpha via functioning as ceRNA for miR199a5p in nonsmall cell lung cancer under hypoxia. Mol Med Rep 17(1):1105-1110

110. Zhang S, Zhang G, Liu J (2016) Long noncoding RNA PVT1 promotes cervical cancer progression through epigenetically silencing miR-200b. Apmis 124(8):649-658

111. McArthur K et al (2011) MicroRNA-200b regulates vascular endothelial growth factor-mediated alterations in diabetic retinopathy. Diabetes 60(4):1314-1323

112. Luengo-Gil G et al (2018) Angiogenic role of miR-20a in breast cancer. PLoS One 13(4):e0194638

113. Tang $\mathrm{Y}$ et al (2018) LncRNAs regulate the cytoskeleton and related Rho/ROCK signaling in cancer metastasis. Mol Cancer 17(1):77

114. Yang $L$ et al (2018) LncRNAs regulate cancer metastasis via binding to functional proteins. Oncotarget 9(1):1426-1443

115. Wei $F$ et al (2018) BPIFB1 (LPLUNC1) inhibits migration and invasion of nasopharyngeal carcinoma by interacting with VTN and VIM. Br J Cancer 118(2):233-247
116. He $\mathrm{R}$ et al (2017) circGFRA1 and GFRA1 act as ceRNAs in triple negative breast cancer by regulating miR-34a. J Exp Clin Cancer Res 36(1):145

117. Wu C et al (2019) Analysis of status and countermeasures of cancer incidence and mortality in China. Sci China Life Sci 62(5):640-647

118. Ma L et al (2010) miR-9, a MYC/MYCN-activated microRNA, regulates E-cadherin and cancer metastasis. Nat Cell Biol 12(3):247-256

119. Cho KB et al (2010) Overexpression of c-myc induces epithelial mesenchymal transition in mammary epithelial cells. Cancer Lett 293(2):230-239

120. Cowling VH et al (2007) c-Myc transforms human mammary epithelial cells through repression of the Wnt inhibitors DKK1 and SFRP1. Mol Cell Biol 27(14):5135-5146

121. Chivukula RR, Mendell JT (2008) Circular reasoning: microRNAs and cell-cycle control. Trends Biochem Sci 33(10):474-481

122. Jackstadt R, Hermeking $H$ (2015) MicroRNAs as regulators and mediators of c-MYC function. Biochim Biophys Acta 1849(5):544-553

123. Ren $\mathrm{H}$ et al (2016) TWIST1 and BMI1 in cancer metastasis and chemoresistance. J Cancer 7(9):1074-1080

124. Selmi A et al (2015) TWIST1 is a direct transcriptional target of MYCN and MYC in neuroblastoma. Cancer Lett 357(1):412-418

125. Zheng X, Hu H, Li S (2016) High expression of lncRNA PVT1 promotes invasion by inducing epithelial-to-mesenchymal transition in esophageal cancer. Oncol Lett 12(4):2357-2362

126. Feng $\mathrm{K}$ et al (2018) Long noncoding RNA PVT1 enhances the viability and invasion of papillary thyroid carcinoma cells by functioning as ceRNA of microRNA-30a through mediating expression of insulin like growth factor 1 receptor. Biomed Pharmacother 104:686-698

127. Wu BQ et al (2017) Long noncoding RNA PVT1 promotes EMT and cell proliferation and migration through downregulating p21 in pancreatic cancer cells. Technol Cancer Res Treat 16(6):819-827

128. Chang Z, Cui J, Song Y (2018) Long noncoding RNA PVT1 promotes EMT via mediating microRNA-186 targeting of Twist1 in prostate cancer. Gene 654:36-42

129. Fan C et al (2017) Role of long non-coding RNAs in glucose metabolism in cancer. Mol Cancer 16(1):130

130. Jiang $X$ et al (2019) Role of the tumor microenvironment in PD-L1/PD-1-mediated tumor immune escape. Mol Cancer 18(1): 10

131. Duan S et al (2019) Natural killer group 2D receptor and its ligands in cancer immune escape. Mol Cancer 18(1):29

132. Tang $\mathrm{Y}$ et al (2017) Linking long non-coding RNAs and SWI/ SNF complexes to chromatin remodeling in cancer. Mol Cancer 16(1):42

133. Mo Y et al (2019) The role of Wnt signaling pathway in tumor metabolic reprogramming. J Cancer 10(16):3789-3797

134. Kim JW et al (2007) Hypoxia-inducible factor 1 and dysregulated c-Myc cooperatively induce vascular endothelial growth factor and metabolic switches hexokinase 2 and pyruvate dehydrogenase kinase 1. Mol Cell Biol 27(21):7381-7393

135. Song J et al (2017) Long non-coding RNA PVT1 promotes glycolysis and tumor progression by regulating miR-497/HK2 axis in osteosarcoma. Biochem Biophys Res Commun 490(2):217-224

136. Chen D et al (2013) Differential effects on ARF stability by normal versus oncogenic levels of c-Myc expression. Mol Cell 51(1):46-56

137. Bond GL, Hu W, Levine AJ (2005) MDM2 is a central node in the p53 pathway: 12 years and counting. Curr Cancer Drug Targets 5(1):3-8 
138. Eischen CM, Boyd K (2012) Decreased Mdm2 expression inhibits tumor development and extends survival independent of Arf and dependent on p53. PLoS One 7(9):e46148

139. Guo J et al (2018) Long noncoding RNA PVT1 modulates hepatocellular carcinoma cell proliferation and apoptosis by recruiting EZH2. Cancer Cell Int 18:98

140. Wan L et al (2016) Long noncoding RNA PVT1 promotes non-small cell lung cancer cell proliferation through epigenetically regulating LATS2 expression. Mol Cancer Ther 15(5):1082-1094

141. Murphy DJ et al (2008) Distinct thresholds govern Myc's biological output in vivo. Cancer Cell 14(6):447-457

142. Wei MC et al (2001) Proapoptotic BAX and BAK: a requisite gateway to mitochondrial dysfunction and death. Science 292(5517):727-730

143. Liu E et al (2015) Overexpression of long non-coding RNA PVT1 in ovarian cancer cells promotes cisplatin resistance by regulating apoptotic pathways. Int J Clin Exp Med 8(11):20565-20572

144. Yang J et al (2017) LncRNA PVT1 predicts prognosis and regulates tumor growth in prostate cancer. Biosci Biotechnol Biochem 81(12):2301-2306

145. Fan CM et al (2019) circMAN1A2 could serve as a novel serum biomarker for malignant tumors. Cancer Sci 110(7):2180-2188

146. Wang Y et al (2017) Circular RNAs in human cancer. Mol Cancer 16(1):25

147. Zhong Y et al (2018) Circular RNAs function as ceRNAs to regulate and control human cancer progression. Mol Cancer 17(1):79

148. Deng $X$ et al (2018) Application of atomic force microscopy in cancer research. J Nanobiotechnol 16(1):102

149. Zhou R et al (2018) Circular RNAs (circRNAs) in cancer. Cancer Lett 425:134-142

150. Panda AC et al (2017) Identification of senescence-associated circular RNAs (SAC-RNAs) reveals senescence suppressor CircPVT1. Nucleic Acids Res 45(7):4021-4035

151. Bussing I, Slack FJ, Grosshans H (2008) let-7 microRNAs in development, stem cells and cancer. Trends Mol Med 14(9):400-409

152. Soucek L et al (2008) Modelling Myc inhibition as a cancer therapy. Nature 455(7213):679-683

153. Salehi M, Sharifi M (2018) Induction of apoptosis and necrosis in human acute erythroleukemia cells by inhibition of long noncoding RNA PVT1. Mol Biol Res Commun 7(2):89-96

154. Ping $\mathrm{G}$ et al (2018) Silencing long noncoding RNA PVT1 inhibits tumorigenesis and cisplatin resistance of colorectal cancer. Am J Transl Res 10(1):138-149
155. Wu D et al (2017) Knockdown of Lncrna PVT1 enhances radiosensitivity in non-small cell lung cancer by sponging Mir-195. Cell Physiol Biochem 42(6):2453-2466

156. You L et al (2011) Genome-wide screen identifies PVT1 as a regulator of Gemcitabine sensitivity in human pancreatic cancer cells. Biochem Biophys Res Commun 407(1):1-6

157. You L et al (2018) Gemcitabine exhibits a suppressive effect on pancreatic cancer cell growth by regulating processing of PVT1 to miR1207. Mol Oncol 12(12):2147-2164

158. Li T, Meng XL, Yang WQ (2017) Long noncoding RNA PVT1 acts as a "sponge" to inhibit microRNA-152 in gastric cancer cells. Dig Dis Sci 62(11):3021-3028

159. Yang Q et al (2018) Long non-coding RNA PVT1 promotes cell proliferation and invasion through regulating miR-133a in ovarian cancer. Biomed Pharmacother 106:61-67

160. Chen $Y$ et al (2018) LncRNA PVT1 promotes ovarian cancer progression by silencing miR-214. Cancer Biol Med 15(3):238-250

161. Xue W et al (2018) PVT1 regulates the malignant behaviors of human glioma cells by targeting miR-190a-5p and miR-488-3p. Biochim Biophys Acta Mol Basis Dis 1864(5 Pt A):1783-1794

162. Ma Y et al (2017) PVT1 affects growth of glioma microvascular endothelial cells by negatively regulating miR-186. Tumour Biol 39(3): 1010428317694326

163. Xiao $\mathrm{M}$ et al (2018) Prognostic values of long noncoding RNA PVT1 in various carcinomas: an updated systematic review and meta-analysis. Cell Prolif 51(6):e12519

164. Zhu S et al (2017) Prognostic value of long non-coding RNA PVT1 as a novel biomarker in various cancers: a meta-analysis. Oncotarget 8(68):113174-113184

165. Chen X et al (2018) lncRNA PVT1 identified as an independent biomarker for prognosis surveillance of solid tumors based on transcriptome data and meta-analysis. Cancer Manag Res 10:2711-2727

166. Liu C et al (2018) Long noncoding RNA PVT1 as a novel predictor of metastasis, clinicopathological characteristics and prognosis in human cancers: a meta-analysis. Pathol Oncol Res 25(3):837-847

167. $\mathrm{MaC}$ et al (2018) Meta-analysis of the prognostic value of long non-coding RNA PVT1 for cancer patients. Medicine (Baltimore) 97(49):e13548

Publisher's Note Springer Nature remains neutral with regard to jurisdictional claims in published maps and institutional affiliations. 